\title{
Rotating Group Design for Vane Pump Flow Ripple Reduction
}

\author{
Leonardo Zanetti-Rocha and Samir N.Y. Gerges \\ Department of Mechanical Engineering, Federal University of Santa Catarina, University Campus, Trindade, \\ 88040-900, Florianpolis-SC, Brazil
}

D. Nigel Johnston

Department of Mechanical Engineering, University of Bath, Bath, BA2 7AY, UK

Jorge P. Arenas

Institute of Acoustics, University Austral of Chile, PO Box 567, Valdivia, Chile

(Received 12 November 2012; accepted 20 August 2013)

It is well known that the hydraulic pump is the main source of noise in hydraulic power steering systems. This noise is produced by the pulsating flow transmitted through the fluid due to the cyclic pumping mechanism. This flow ripple and pressure ripple spread through the hydraulic circuit, interacting in a complex way with other parts of the vehicle. This process generates annoying audible noise inside the vehicle. This work addresses a new approach to flow ripple reduction by tuning the pump rotating group. The method consists of making the outlet flow as steady as possible by modifying the rotating group geometry. A MATLAB/Simulink-based pump model has been created according to the new geometrical characteristics and their numerical results, compared to the regular pump ones. In addition, a flow ripple experiment was conducted using the secondary source method (SSM to validate the numerically-predicted results of the regular pump. The results of the new design show significant amplitude reduction of the flow ripple amplitudes at different operating conditions. In particular, the flow ripple reduction at the first harmonic is almost $20 \mathrm{~dB}$ when the pump runs in a parking manoeuvres operating condition (1000 rpm at 50 bar of backpressure).

\section{INTRODUCTION}

Noise produced by the hydraulic power steering pump when operating a car can, in some situations, be perceived inside the vehicle as an annoying sound. The flow variations (flow ripple) generated by the pump through its natural operating process are transmitted along the power steering hydraulic circuit, interacting with the impedance of the hoses and tubes. This process generates structural vibration and, consequently, sound emission around the circuit. ${ }^{1}$

In some vehicles, the hydraulic circuit is tuned in order to avoid hearing noise inside the cabin. Flexible hoses, sometimes with tuning cables, are usually used to increase circuit compliance in order to remove unwanted resonance frequencies or reduce the flow ripple amplitude. In fact, there are several techniques and devices-such as silencers, side branches, accumulators, etc.- that can be used to reduce fluid-borne noise along the propagation circuit. ${ }^{2,3}$

However, if a pump can be manufactured with a lower noise profile, this may create a solution that is independent of the hydraulic circuit and does not require costly and time-consuming tuning of the system to achieve the required noise reduction.

The present work is aimed at vane pumps and proposes a new rotating group design, intended to reduce the flow ripple amplitude. Because this new design is not dependent on the number of vanes, an eight vane rotating group was developed to combine cost reduction with flow ripple reduction.
In order to achieve those results, a MATLAB/Simulinkbased numerical model was created from a regular pump design and validated through the experimental secondary source mMethod (SSM), which has been developed at the University of Bath., ${ }^{4,5}$ The SSM has been used as the basis for an international standard to measure pump pressure ripple characteristics. ${ }^{6}$ The simulated results of the new design are compared here with the regular pump ones. A comparison is then made in terms of the flow ripple amplitudes in the frequency domain.

\section{REGULAR PUMP CHARACTERISTICS}

The regular automotive pump has two discharge ports located diametrically opposite to each other, i.e., they are spaced by an angle of $180^{\circ}$ regarding the centre of the thrust plate as the centre of the reference circumference. This type of positive-displacement pump is called a balanced pump because these two opposite ports balance the forces acting against the rotor, and all the walls of the chamber as well, reducing the shaft pump oscillations and overall vibrations. Figure 1 shows the rotating group components and its assembly scheme.

The pump uses the engine torque applied to its shaft to pump oil from the inlet port to the outlet port. Figure 2 shows a scheme of the pumping process inside the pump. The system back pressure varies according to the outlet hydraulic circuit constraint, increasing the pressure as the constraint increases. 